\title{
Medical Image Denoising Via Matrix Norm Minimization Problems
}

\author{
Zainab Abd-Alzahra* and Basad Al-Sarray
}

Computer science Department, College of Science, University of Baghdad, Baghdad, Iraq

\begin{tabular}{ll}
\hline \multicolumn{1}{c}{ Article's Information } & \multicolumn{1}{c}{ Abstract } \\
\hline $\begin{array}{l}\text { Received: } \\
\text { 18-01-2021 }\end{array}$ & This paper presents the matrix completion problem for image denoising. Three \\
Accepted: & problems based on matrix norm are performing: Spectral norm minimization \\
problem (SNP), Nuclear norm minimization problem (NNP), and Weighted & nuclear norm minimization problem (WNNP). In general, images representing \\
Published: & by a matrix this matrix contains the information of the image, some information \\
$27-06-2021$ & is irrelevant or unfavorable, so to overcome this unwanted information in the \\
Keywords: & image matrix, information completion is used to comperes the matrix and \\
Image De noising & remove this unwanted information. The unwanted information is handled by \\
Matrix completion & defining 0,1 -operator under some threshold. Applying this operator on a given \\
Low rank & matrix keeps the important information in the image and removing the \\
Weighted nuclear norm & unwanted information by solving the matrix completion problem that is defined \\
Spectral norm & by P. The quadratic programming use to solve the given three norm-based \\
& minimization problems. To improve the optimal solution a weighted exponential \\
& is used to compute the weighted vector of spectral that use to improve the \\
& threshold of optimal low rank that getting from solving the nuclear norm and \\
& spectral norm problems. The result of applying the proposed method on different \\
& types of images is given by adopting some metrics. The results showed the \\
& ability of the given methods. \\
\hline
\end{tabular}

DOI: $10.22401 /$ ANJS.24.2.10

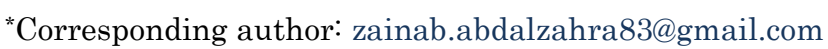

\section{Introduction}

Low-rank matrix approximation is used for recovering the low-rank matrix from its degraded data points. It has been used in various applications of computer vision. The lowrank models were applied successfully for image restoration problems. In some problems, the low-rank is used as a global prior, and defining image restoration as a low-rank problem directly. Matrix approximation for rank reduction is used significantly in signal and computer vision/image processing, in image compression low-rank is used to get the redundant information; here low-rank matrices give a better compressed. Low-rank matrix completion is an NP-hard problem so, most of the time the problems are derived to be heuristic problems based on some methods of relaxation to find an approximate solution. Sparse in image studied at an early stage of image denoising research. To overcome the sparse in the image a shrinkage or optimization approaches have been suggested to find the effect of sparse coefficients in the transformation domain. During the last several decades, many attempts have been made to find more appropriate transformation domains as well as sparse measurements for image denoising. Cai et al. in [1] introduced a novel algorithm to approximate the matrix with the minimum, the nuclear norm on convex constraints, the problem derived to be a convex relaxation of a rank minimization. In [2], they studied NP-hard weights and regularization for weighted low-rank approximation with constant rank. They derived the regularized version by parameterizing bounds in by statistical dimension. Mazumder et al. in [3] presented convex relaxation techniques of the regularized low-rank problems for large-scale matrix completion problems. They use the nuclear norm as regularization; they provide a convex algorithm for minimizing the reconstruction error subject to bound on the nuclear norm. Their algorithm is SOFT-IMPUTE iteratively replaces the missing elements with those obtained from a soft-threshold SVD. Agaian et al. in [4] presented a noise detection and reduction scheme employing a fast logical (binary) transform-based Boolean minimization algorithm which is capable of de-noising both binary and multivalued images corrupted by impulse noise. Xie et al. in [5] proposed a weighted Schatten p-Norm Minimization model for image denoising. in [6] proposed a speed-up technique for the non-local means (NLM) image denoising method supported local binary descriptors. $\mathrm{Gu}$ et al. in [7] proposed a weighted nuclear norm minimization model to adaptively regularize the singular value of the matrix.

The rest of the paper contains section 2 which is a theoretical background, while section 3 presents the problem formulation, and section 4 gives the proposed method. Finally, section 5 gives the results. 


\title{
Al-Nahrain Journal of Science
}

\author{
ANJS, Vol.24 (2), June, 2021, pp. 72-77
}

\section{Theoretical Background}

Matrix completion is a crucial problem that has been researched broadly in recent times. MC-problem varies from the problem of matrix approximation by the fact that the known entries must remain fixed while changing their role from the objective function to be minimized to a part of the constraint. A well-researched MC-problem appears as a problem of rank minimization. Because rank minimization is not convex and NP-hard, it is generally relaxed for the nuclear norm minimization, though other constraints are often used like spectral norm minimization. The problem is: min $\operatorname{rank}(X)$

Subject to:

$$
X_{i, j}=L_{i, j},(i, j) \in \Omega
$$

it is often approximated by: $\min \|X\|_{*}$

Subject to:

$$
X_{i, j}=L_{i, j},(i, j) \in \Omega
$$

where $\Omega$ is the set of indices $(i, j)$ that are not equal to zero, $X_{i, j}$ is an original matrix, $L_{i, j}$ is a noised matrix, $\|X\|_{*}$ is the nuclear norm of $X$ which is a singular values summation of $X$. A little value of $\|X\|_{*}$ is getting from the low-rank property [3]. A solution that iterative, which is a singular value threshold basis is given in [1].

Recently the nuclear norm minimization (NNM) has a lot of applications in computer vision and machine learning research. The standard nuclear norm of a matrix $X \in R^{m \times n}$ is defined as the sum of all its singular values $\sigma_{i}(X), i=1,2, \ldots, \min (n, m)$ and represented by $\|X\|_{*}=$ $\sum_{i}\left|\sigma_{i}(X)\right|$. Nuclear norm is used to define a convex relaxation of the rank penalty of a matrix.

The standard NNM problem used to find approximate solution matrix $\mathrm{L}$ of $\mathrm{X}$ by solving a minimization problem

$$
\min \|X-L\|_{F}^{2}+\lambda\|L\|_{*}
$$

where $\lambda$ is a regularization parameter. The nuclear norm minimization problem has a solution equal to $L^{*}=$ $U S_{\lambda}(\Sigma) V^{T}$, where $X=\mathrm{U} \Sigma V^{T}$ is the SVD of $X$ and $S_{\lambda}(\Sigma)=\max (0, \Sigma-\lambda / 2)$.

However, some entries that participate have a role within the process, the solution is sufficient for finding the approximation.

\section{Problem Formulation}

To detecting and removing the unwanted information, define un operator $P$ to be

$$
P=\left\{\begin{array}{cc}
1 & \text { if } X_{i, j}>S_{\text {thr }} \\
0 & \text { otherwise }
\end{array}\right.
$$

Let $\mathcal{O} \in R^{m \times n}$, with $\{0,1\}$ entries that computing form $P$, and $P X=X \otimes \mathcal{O}$, so $P L=L \otimes \mathcal{O}$, then Equation (3) reformed by:

$$
\text { opt }=\operatorname{argmin}\|P X-P L\|_{F}^{2}
$$

Subject to

$$
\|P L\|_{*}<=r
$$

In this paper, three types of matrix norm-based problems are derived based on $\{0,1\}-P$. Because of convexity, the solution is globally optimal. This study presents three nuclear norm-based problems.

The nuclear norm problem (NNP) is:

$$
\text { opt }=\min \|P X-P L\|_{F}^{2}
$$

Subject to:

$$
\|\mathrm{P} L\|_{*} \leq \mathcal{Q}_{1} .
$$

The constraint here is rank of $X$, initially set $r=$ $\min \operatorname{dim}(X)$.

Spectral norm problem (SNP):

opt $=\operatorname{argmin}\|P X-P L\|_{F}^{2}$

Subject to:

$$
\|\mathrm{P} L\|_{2} \leq Q_{2} \text {. }
$$

$\delta$, depend on the maximum value of the spectral vector, [7].

Nuclear Weighted problem (WNNP) derived to improve the flexibility of NNM. For any $X \in \mathbb{R}^{m \times n}$, choose a diagonal non-negative matrix $W \in \mathbb{R}^{m \times n}$ with non-ascending ordered diagonal elements [7,11]. The weighted nuclear norm of $X$ is

$$
\|X\|_{W *}=\sum_{i}\left|w_{i} \sigma_{i}(X)\right|
$$

Then Equation (5) defined to be

$$
\text { opt }=\operatorname{argmin}\|P X-P L\|_{F}^{2}
$$

Subject to:

$\|\mathrm{P} L\|_{\mathrm{W}, *} \leq \mathrm{Q}_{3}$.

$\mathrm{Q}_{i}$ improved based on the sequence of steps in the proposed algorithm.

\section{Proposed Methods}

This work presents some techniques for solving quadratic programming with form

$$
\min \frac{1}{2}\|X-\hat{X}\|_{F}^{2}+F(X)
$$

Subject to:

$$
F(X) \leq \delta
$$

Equation (10) solved by adding some assumptions on the set of lower and upper bounds on the design variables which are $r$-rank $(L)$ in equation $(6), \max \left(\sigma_{i}(X)\right)$ in equation (7).

\subsection{Weighted majority for spectral selection}

Nuclear norm regularizes use all singular values directly this method of using spectral vector is not sufficient enough to use the affected singular values. So, we presented a method that assigned weights to different singular values. Thus, in this work, we adopted Weighted Majority Algorithm (WMA) for spectral selection [9][10]. The scheme of WMA given by Littlestone and Warmuth (1994), starting by assigning probabilities $p_{t}=$ $\left(p_{t}^{1}, \ldots, p_{t}^{K}\right)$ to $\mathrm{K}$ 's actions. Actions get loss $l_{t} \in[0,1]^{K}$. The aim is to minimize the regret

$$
\text { Regret }_{T}=\sum_{t=1}^{T} p_{t} l_{t}-L_{T}^{*}
$$
where $L_{T}^{*}=\min _{k} \sum_{1}^{T} l_{t}^{k}$. 


\title{
Al-Nahrain Journal of Science
}

\author{
ANJS, Vol.24 (2), June, 2021, pp. 72-77
}

\section{Weighted Majority Algorithm:}

Initialization: Fix an $\eta$ with each expert $i$, associate the weight $\psi_{i}^{t}=1$.

For $t=1$ to $T$ make the prediction that is the weighted majority of the experts' predictions based on the weights $\psi_{1}^{t}, \ldots, \psi_{n}^{t}$.

For every expert, $i$ who predicts wrongly, decrease his weight for the next round by multiplying it by a factor of $1-\eta, \psi_{i}^{t+1}=(1-\eta) \psi_{i}^{t}$

Here, Weighted Exponential (WE) are used as a version of WMA, applied on computing the constraint of problems in Equations (6), (7) and (9), were WE is applied on the sigmoid vector of the optimal low rank at each iteration for the mean loop.

The approach consists of computing a vector $\psi^{\tau}=$ $\left(\psi_{1}^{\tau}, \ldots, \psi_{n}^{\tau}\right)$ of weights for $\tau=n+1, n+2, \ldots, n+1+$ $T$. Using these weights, one assigns to each model the probability (compute prediction).

$$
p_{\tau}=\frac{\sum_{i=1}^{N} \psi_{i}^{\tau} l_{i}^{\tau}}{\sum_{i=1}^{N} \psi_{i}^{\tau}}
$$

The goal is to learn from the data the best probabilities to assign. For this purpose, define a loss function at time $\geq K+1$

$$
l_{\tau}(p)=\sum_{i=1 \ldots K}^{\min (i, j)} p_{i}\left(\sigma_{i}(X)-\sigma_{i}(L)\right)^{2}
$$

An interesting criterion to optimize is the cumulated loss

$$
L_{T o t}(p)=\sum_{t=n+1}^{n+1+T} l_{\tau}(\pi)
$$

The Exponential Weighting Algorithm is a method often used in computer science, machine learning, and statistics to optimize the cumulated loss. In this method, the weights are updated using the following very simple rule

$$
\psi_{i}^{\tau+1}=\psi_{i}^{\tau} \exp \left(-\eta \sum\left(X_{i j}-L_{i j}\right)^{2}\right)
$$

The approach we adopt here is standard except for the fact that that the loss function is unbounded. As is well known, this method can be seen as an instance of the Mirror Descent Method for finding an optimal linear aggregation of predictors using proximal iterations based on the entopic Kulback-Leibler divergence, more details see $[9,10]$.

\subsection{Proposed algorithm}

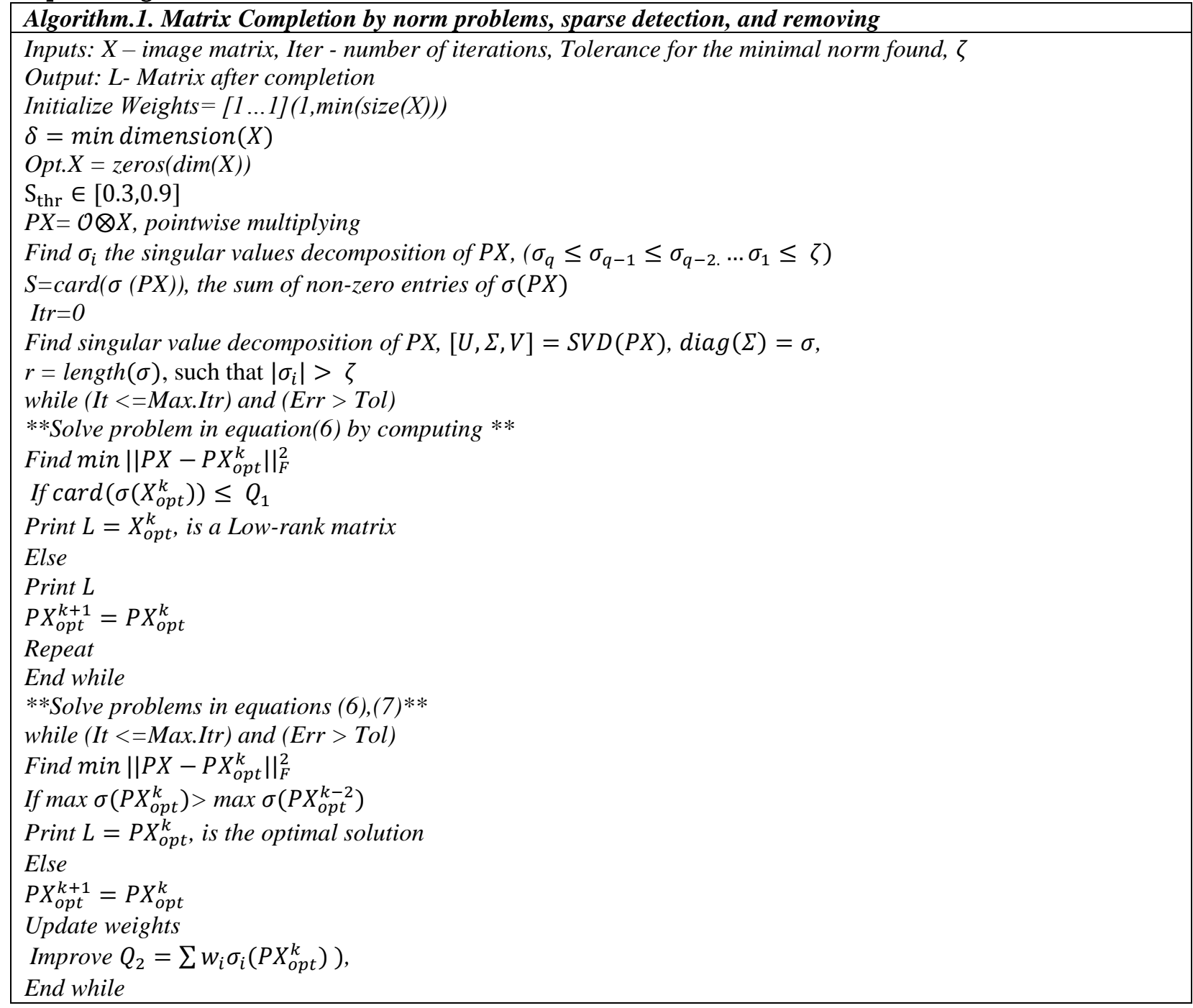




\section{Al-Nahrain Journal of Science \\ ANJS, Vol.24 (2), June, 2021, pp. 72-77}

\section{Results}

The proposed techniques used for solving constraint problems Equations (6), (7) and (9) are implemented for images denoising. Images are used study is medical infarct scan images, format: LLVS, for human head data-set The PCCV Project: Benchmarking Vision Systems. The number of iterations is 150 , Tolerance Tol $=0.0001$, upper bound for the $\operatorname{rank}(X)=2 \times \min (\operatorname{dimensions}$ of $X$. The threshold $S_{\text {thr }}$ for detecting the unwanted information is taking equal to $\{0.3,0.7,0.9\}$.
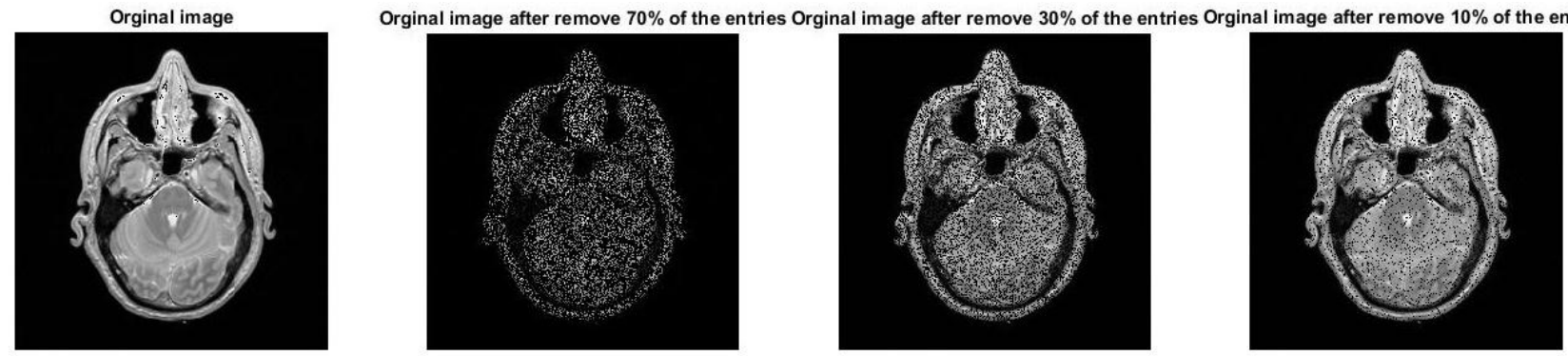

Figure.1.a. Image. 1 detecting the unwanted information with the threshold $S_{t h r}$ equal to $\{0.3,0.7,0.9\}$, respectively.
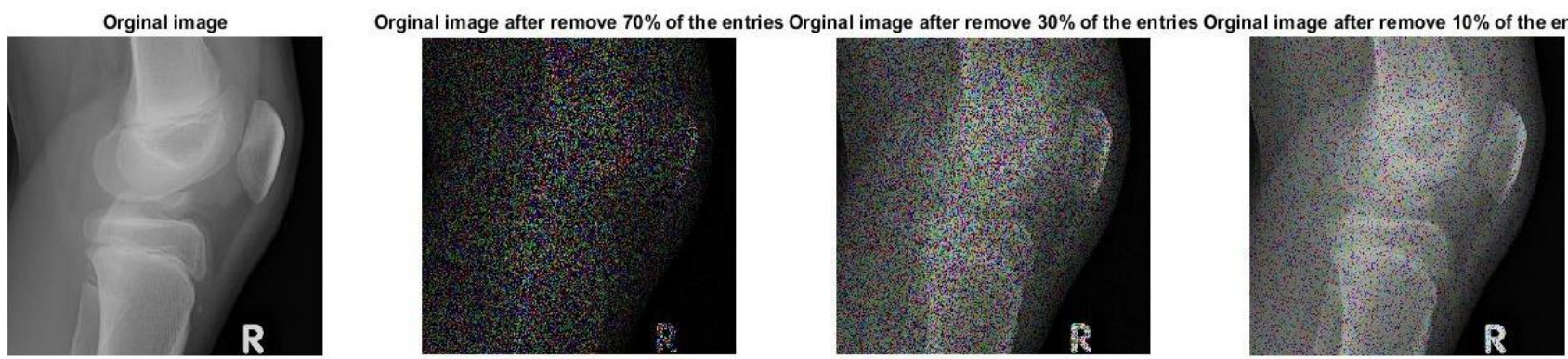

Figure.1.b. Image. 1 detecting the unwanted information with the threshold $S_{\text {thr }}$ equal to $\{0.3,0.7,0.9\}$, respectively.
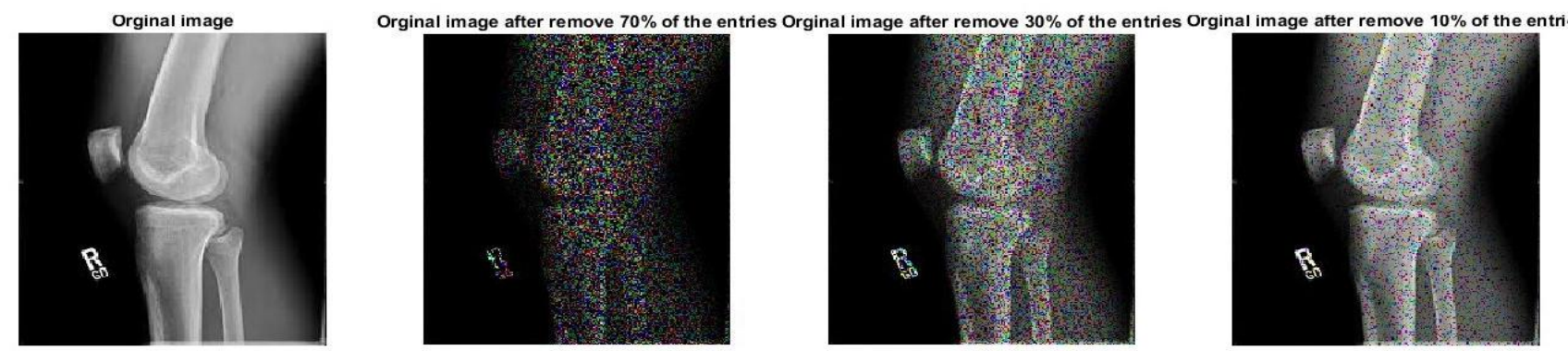

Figure.1.c. Image. 3 detecting the unwanted information with the threshold $S_{t h r}$ equal to $\{0.3,0.7,0.9\}$, respectively. 


\section{Al-Nahrain Journal of Science}

ANJS, Vol.24 (2), June, 2021, pp. 72-77

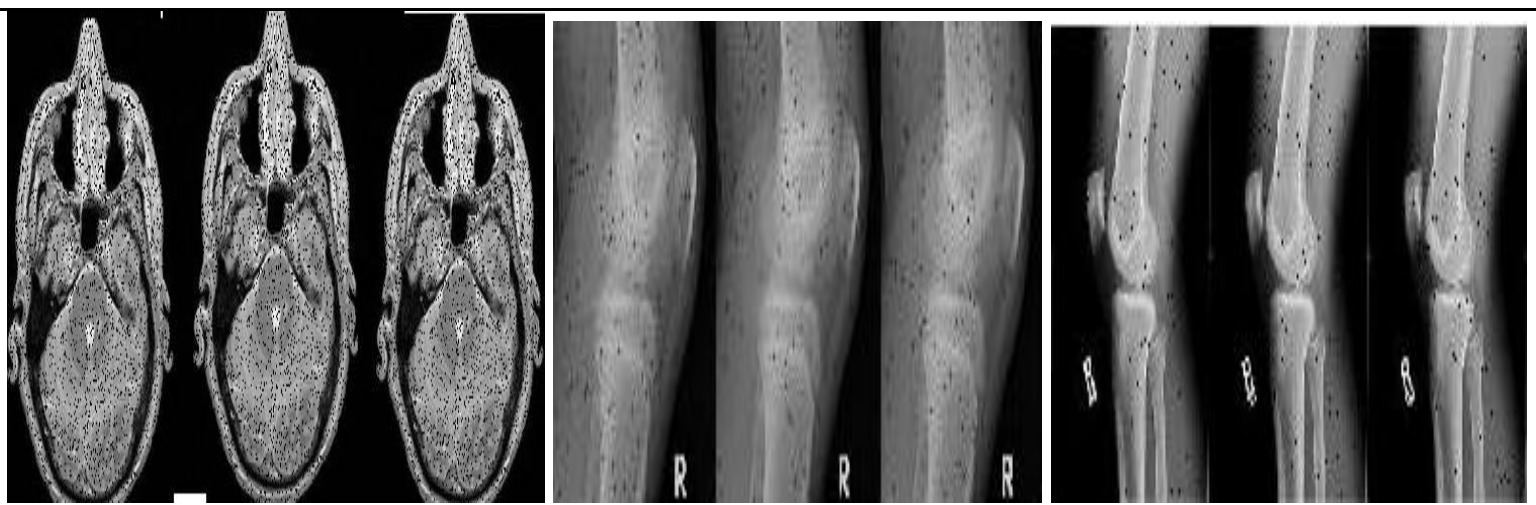

Figure.2.a. Image denoising after removing $10 \%$ of entries based on the optimization of norm problems.

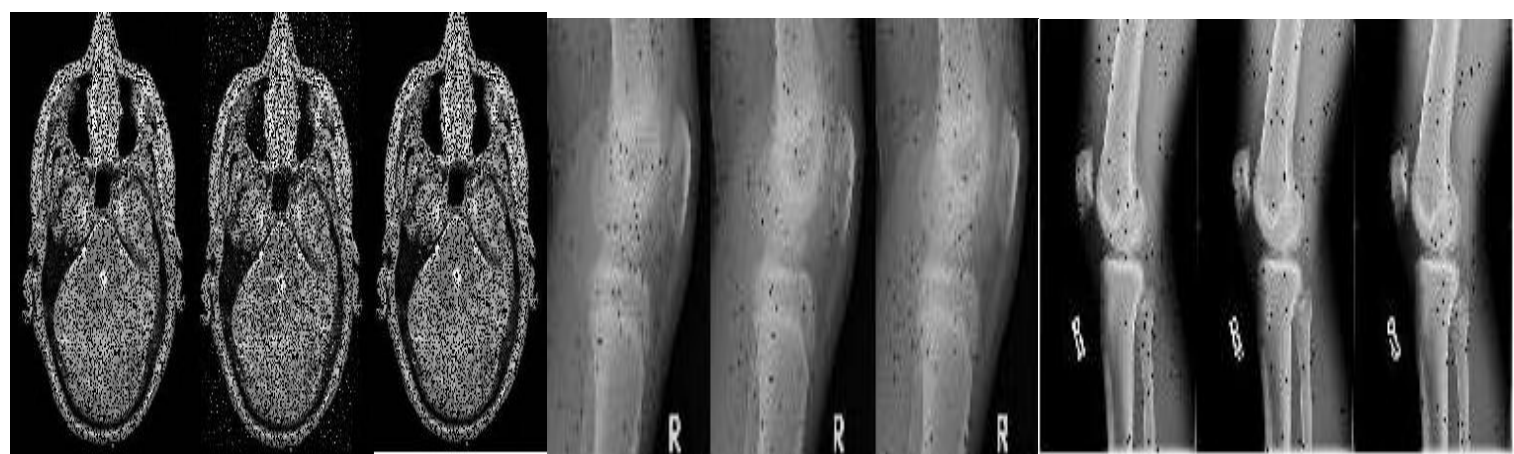

Figure.2.b. Image denoising after removing $30 \%$ of entries based on the optimization of norm problems.

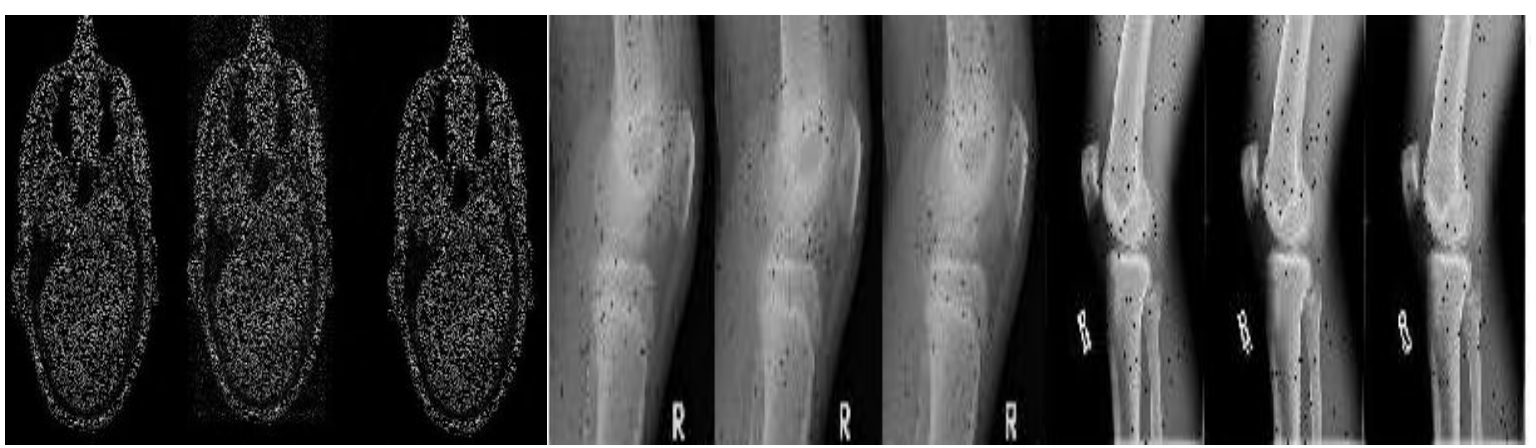

Figure.2.c Image denoising after removing $70 \%$ of entries based on optimization of norm problems.
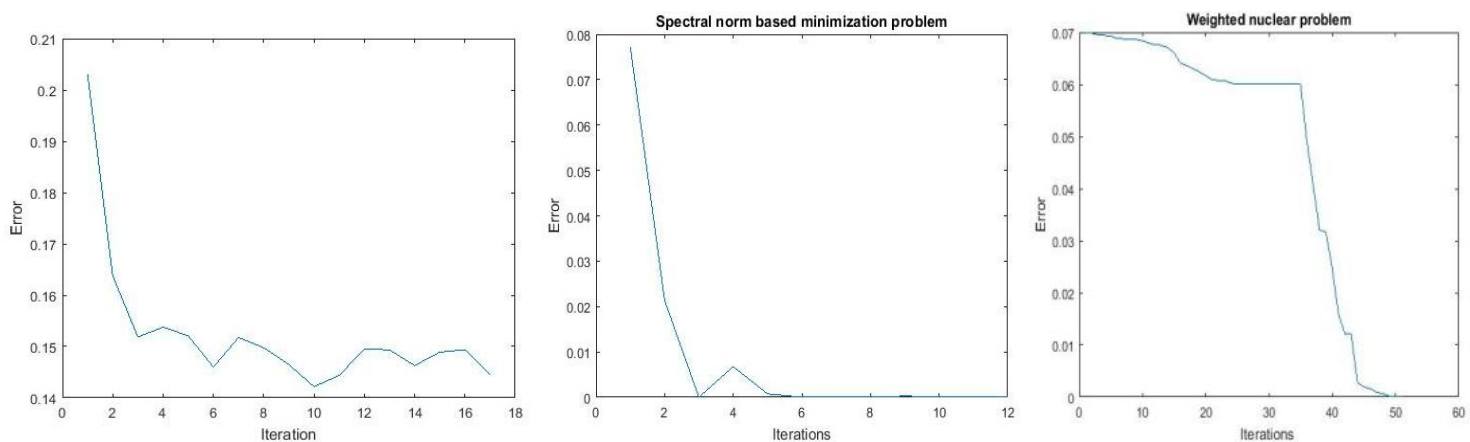

Figure.3. Error function for Image.1 denoising via norm minimization problems concerning the number of iterations.

Metrics used in this work are Mean Square Error (MSE), Structure Similarity Index Measurement (SSIM), Peak Signal to Noise Ratio (PSNR). The results are given in the figures and tables given below. The Table compared the metrics values for image denoising via nuclear norm minimization problem. From the table, it is clear that for the SNP method the mean square error (MSE) is minimum and the peak signal to noise ratio (PSNR) is maximum, also the contrast to noise ratio (SSIM) is maximum. 


\section{Al-Nahrain Journal of Science}

ANJS, Vol.24 (2), June, 2021, pp. 72-77

Table.1. Metrics for image denoising via norm minimization problem.

\begin{tabular}{||c|c|c|c|c||}
\hline \hline Test image & Problem & MSE & SSIM & PSNR \\
\hline \hline & NNP & 13.9789932387 & 0.3008295856 & 38.4754833366 \\
Image.1 & SNP & 11.6917431405 & 0.3430439156 & 67.4513613309 \\
& WNNP & 34.6718689902 & 0.0582616502 & 21.6711406269 \\
\hline \multirow{4}{*}{ Image.2 } & NNP & 13.2329122514 & 0.1966175636 & 40.5205328103 \\
& SNP & 10.8150681727 & 0.22582127297 & 62.850227302 \\
& WNNP & 15.7053804848 & 0.20357968744 & 23.8621894609 \\
\hline & NNP & 14.0497183302 & 0.19254688105 & 39.2076076227 \\
Image.3 & SNP & 11.5440852244 & 0.24512938813 & 67.6426368185 \\
& WNNP & 17.5184296129 & 0.02960063119 & 24.1146699723 \\
\hline \hline
\end{tabular}

\section{Conclusions}

This paper presents matrix completion for the image denoising problem. In general, noises in image getting from unwanted information that found in the image, in this work the unwanted information is determined by defining an operator we call it $\{0,1\}-P$ and define the original matrix to be the point-wise multiply $X=P X .\{0,1\}-P$ applied on deriving a convex constrained minimization problems known as matrix compilation. Three matrix compilation problem minimization problems are derived: minimization problem-based nuclear norm, minimization problem-based spectral norm, minimization problem based on the weighted nuclear norm. These problems are derived to be constrained problems, the constraints defined by the spectral norm of the image matrix, here, the efforts are focusing on controlling the lower bound of constraint value $\delta$ by using a majority weighted algorithm to control the spectral vector.

Three types of norm-based problems are given to find the optimal low-rank matrix by applying them to selected medical images. Metrics that used to measures the efficiency of the given method shows the reliability of the method to get a low-rank matrix.

\section{References}

[1] Cai; Jian F.; Emmanuel J. C. and Zuowei S.; "A singular value thresholding algorithm for matrix completion", SIAM Journal on optimization, 20(4), 1956-1982, 2010.

[2] Srebro N. and Tommi J.; "Weighted low-rank approximations", Proceedings of the $20^{\text {th }}$ International Conference on Machine Learning (ICML-03), 2003.

[3] Mazumder R.; Trevor H. and Robert T.; "Spectral regularization algorithms for learning large incomplete matrices", The Journal of Machine Learning Research 11, 2287-2322, 2010.

[4] Agaian S. S.; Thomas A. B. and Karen A. P.; "Denoising of images using logical (binary) transforms", Visual Information Processing XIII, International Society for Optics and Photonics; 5438, 2004.

[5] Xie Y.; et al.; "Weighted Schatten p-norm minimization for image denoising and background subtraction", IEEE transactions on image processing, 25(10), 4842-4857, 2016.

[6] Yu H. and Li A.; "Real-Time Non-Local Means Image Denoising Algorithm Based on Local Binary Descriptor", KSII Transactions on Internet \& Information Systems, 10(2), Feb 1, 2016.

[7] $\mathrm{Gu} \mathrm{S}$; ; et al.; "Weighted nuclear norm minimization with application to image denoising", Proceedings of the IEEE conference on computer vision and pattern recognition; 2014.

[8] Gu S.; et al.; "Weighted nuclear norm minimization and its applications to low-level vision", International Journal of computer vision, 121(2), 183-208, 2017.

[9] Littlestone N.; Manfred K. W.; "The weighted majority algorithm", Information and computation, 108(2), 212$261 ; 1994$.

[10] Shalev-Shwartz S.; "Online learning and online convex optimization", Foundations and trends in Machine Learning, 4(2); 107-194; 2011.

[11] Xie Q.; Deyu M.; Shuhang G.; Lei Z.; Wangmeng Z.; Xiangchu F. and Zongben X.; "On the optimal solution of weighted nuclear norm minimization", arXiv preprint arXiv, 1405-6012, 2014. 